\title{
Fruit Disease Classification based on Texture Features
}

\author{
Ranjit K. N. \\ Department of Computer Science \\ \& Engineering \\ HKBK Engineering College, \\ Bangaluru, Karnataka, India
}

\author{
Naveen C. \\ Department of Computer Science \\ \& Engineering \\ HKBK Engineering College, \\ Bangaluru, Karnataka, India
}

\author{
Chethan H. K. \\ Department of Computer Science \\ \& Engineering \\ Maharaja Institute of Technology, \\ Mysuru, Karnataka, India
}

\begin{abstract}
Nowadays digitization and automation of machine in agriculture field plays prominent role. In this paper, we have proposed method to classify fruit as diseased and nondiseased. Firstly, we used K means clustering method for segmentation of diseased regions. Later, we used to extract texture features on segmented diseased regions. We have collected fruit diseased images from internet to create dataset and totally we have collect 2500 images from 10 fruit classes. We have conducted extensive experimentation using Probabilistic Neural Network and results shows that proposed method gives better performance compared to SVM and KNN.
\end{abstract}

\section{Keywords}

Fruit disease, K-Means, Texture, LBP, Neural Network

\section{INTRODUCTION}

Agriculture is the mother of all cultures. It has played a key role in the development of human civilization. Agricultural production system is an outcome of a complex interaction of soil, seed and agro chemicals (including fertilizers). Therefore, there is need to take good judgment for management of all the inputs is essential for the sustainability of a complex system. The focus on increasing the productivity, without considering the ecological impacts has resulted into environmental degradation. Diseases and insect pests are the major problems in agriculture. These require careful diagnosis and timely handling to protect the crops from heavy losses. Fruit diseases can cause significant losses in yield and quality appeared in harvesting. For example, soybean rust (a fungal disease in soybeans) has caused a significant economic loss and just by removing $20 \%$ of the infection, the farmers may benefit with an approximately 11 million-dollar profit (Roberts et al., 2006). Some fruit diseases also infect other areas of the tree causing diseases of twigs, leaves and branches. An early detection of fruit diseases can aid in decreasing such losses and can stop further spread of diseases. In plant, diseases can be found in various parts such as fruit, stem and leaves. In development of India there is major contribution of agricultural field. There are so many fruits like apple, oranges, mango and grapes etc. that are exported from India and give more profit to the farmers. Diseases in fruit cause devastating problem in economic losses and production in agricultural industry worldwide. Generally the naked eye method is used to identify the diseases. In this method experts are involved who have the ability to detect the changes in leaf color. This method involves lots of efforts, takes long time and also not practical for the large fields. Many times different experts identify the same disease as the different disease. This method is expensive as it requires continuous monitoring of experts. Apple fruit diseases can cause major losses in yield and quality appeared in harvesting. To know what control factors to take next year to avoid losses, it is crucial to recognize what is being observed. Some common diseases of apple fruits are apple scab, apple rot, and apple blotch [5], Apple scabs are gray or brown corky spots. Apple rot infections produce slightly sunken, circular brown or black spots that may be covered by a red halo. Apple blotch is a fungal disease and appears on the surface of the fruit as dark, irregular or lobed edges.

\section{RELATED WORK}

Recently, a lot of activity in the area of fruit disease detection can be seen in which defect segmentation of fruits are performed using simple threshold approach[1],[2],. An adaptive threshold method for defect segmentation on apples is presented in [3]. Pixels are classified into different classes using different classification methods. Kleynen et al. [4] is based on Bayesian classification where defected or healthy pixels are classified by comparing them with pre-calculated model. In [5], Ojala et al. uses uniform local binary pattern to classify static as well as dynamic textures. Local binary pattern is very efficient feature to define local image pattern. Recent development in agriculture technology has led to a demand of automated non-destructive methods of fruit disease detection. It is desirable that the fruit disease detection tool should accurately classify particular disease. The disease monitoring approaches like spectroscopic and imaging techniques have been used to detect diseases. Current research activities are towards the development of such technologies that can be used for large-scale detection of disease in fruits. Some of the spectroscopic and imaging techniques that are used for the detection of diseases are: fluorescence imaging used by Bravo et al. [6]; Moshou et al. [7]; Chaerle et al. [8], multispectral or hyperspectral imaging used by Moshou et al. [9]; Shafri and Hamdan [10]; Qin et al. [11], infrared spectroscopy used by Spinelli et al. [12]; Purcell Detection and Classification of Fruit Disease : A Review International Research Journal of Engineering and Technology (IRJET) eISSN: 2395 -0056 Volume: 03 Issue: 03 | Mar-2016 www.irjet.net p-ISSN: 2395-0072 (C) 2016, IRJET | Impact Factor value: 4.45 | ISO 9001:2008 Certified Journal | Page 727 et al. [13], fluorescence spectroscopy used by Marcassa et al. [14]; Belasque et al. [15]; Lins et al. [16], visible/multiband spectroscopy used by Yang et al. [17]; Delalieux et al. [18]; Chen et al. [19], and nuclear magnetic resonance (NMR) spectroscopy used by Choi et al. [20]. Hahn [21] reviewed multiple methods (sensors and algorithms) for pathogen detection, with special emphasis on postharvest diseases. Several techniques for detecting diseases is reviewed in [22] such as, Molecular techniques, Spectroscopic techniques (Fluorescence spectroscopy and Visible and infrared spectroscopy), and Imaging techniques (Fluorescence imaging and Hyper-spectral imaging). In [7], a ground-based real-time remote sensing system for detecting diseases in arable crops in an early stage of disease development is developed. The authors have used an imaging spectrograph with hyper-spectral reflection images of infected and 
simultaneously they have also used multi-spectral fluorescence images using UV-blue excitation on the same specimen. In [8], the authors have used fluorescence imaging and compared it to the visual development of diseases of tobacco mosaic virus infection in resistant tobacco. Large scale plantation of oil palm trees requires on-time detection of diseases as the ganoderma basal stem rot disease was present in more than in Peninsular Malaysia 50\% of the oil palm plantations. To deal with this problem, airborne hyperspectral imagery offers a better solution [10] in order to detect and map the oil palm trees that were affected by the disease on time. Citrus canker is among the most devastating diseases that affect marketability of citrus crops. In [11], a hyperspectral imaging approach is developed for detecting canker lesions on citrus fruit and hyper-spectral imaging system is developed for acquiring reflectance images from citrus samples in the spectral region from 450 to $930 \mathrm{~nm}$. In [13], the authors have investigated the power of NIR spectroscopy as an alternative to rate clones of sugarcane leaf spectra from direct measurement and examined its potential using a calibration model to successfully predict resistance ratings based on a chemometrics approach such as partial least squares. Marcassa et al. [14] have applied laser-induced fluorescence spectroscopy to investigate biological processes in orange trees. They have investigated water stress and Citrus Canker, which is a disease produced by the Xanthomonas axonopodis pvcitri. Lins et al. [16] have developed an optical technique to detect and diagnose citrus canker in citrus plants with a portable field spectrometer unit. In [18], the authors have investigated the use of hyper-spectral methods caused by apple scab for early detection of plant stress to move towards more reduced and efficient application of fertilizers, pesticides or other crop management treatments for the apple orchards.

\section{PROPOSED METHOD}

In this section, first part shows how the method segment the diseased area using K-Means clustering and second part describes the different textures features extraction on segmented diseased area. Then feed the feature vector to Probabilistic Neural network and also compared with the traditional classifiers.

\subsection{Image Segmentation}

K-Means clustering technique is used for the image segmentation. K-Means clustering algorithm was developed by J. MacQueen (1967). K-means is generally used to determine the natural groupings of pixels present in an image. It is attractive in practice, because it is straightforward and it is generally very fast [1]. It partitions the input dataset into $\mathrm{k}$ clusters in which one cluster contains the majority of the diseased part of the image (in this approach we partitions the image into three clusters). Each cluster is represented by an adaptively changing center, starting from some initial values named seed-points. K-means clustering computes the distances between the inputs (also called input data points) and centers, and assigns inputs to the nearest center. The introduced framework of defect segmentation operates in six steps as follows,

\section{Step 1. Read the input image of defected fruit.}

Step 2. Transform Image from RGB to L*a*b* Color Space. We have used $\mathrm{L}^{*} \mathrm{a} \mathrm{b}^{*}$ color space because it consists of a luminosity layer in ' $\mathrm{L}^{*}$ 'channel and two chromaticity layer in 'a*' and 'b*e channels. Using $\mathrm{L}^{*} \mathrm{a} \mathrm{b}^{*}$ color space is computationally efficient because all of the color information is present in the ' $a *$ ' and ' $b{ }^{* '}$ layers only.
Step 3. Classify Colors using K-Means Clustering in ' $a * b *$ Space. To measure the difference between two colors, Euclidean distance metric is used.

Step 4. Label Each Pixel in the Image from the Results of KMeans. For every pixel in our input, K-means computes an index corresponding to a cluster. Every pixel of the image will be labeled with its cluster index.

Step 5. Generate Images that Segment the Input Image by Color. We have to separate the pixels in image by color using pixel labels, which will result different images based on the number of clusters.

Step 6. Select the segment containing disease. In this experiment input image are partitioned into three segments. Fig. 1 demonstrates the output of K-Means clustering for an apple fruit infected with apple rot disease.
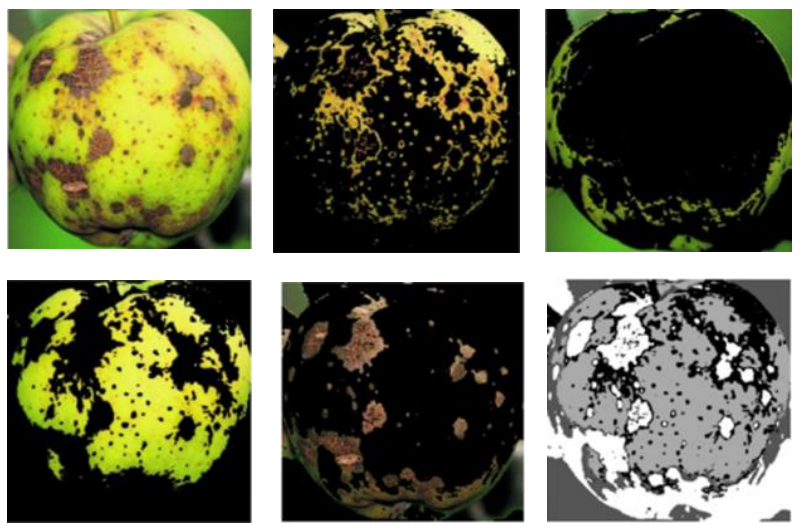

\subsection{Feature Extraction}

In the proposed approach, we have used some state of the art color and texture features to validate the accuracy and efficiency. The features used for the apple fruit disease classification problem are Global Color Histogram, Color Coherence Vector, Local Binary Pattern, and GLCM features.

1) Global Colour Histogram (GCH) The Global Color Histogram $(\mathrm{GCH})$ is the simples approach to encode the information present in an image [16]. The goal is to calculate the HSV global histograms for all the images [17].

2) Color Coherence Vector (CCV) An approach to compare images based on color coherence vectors are presented in [18]. Color histograms are used to compare images in many applications. Their advantages are efficiency, and sensitivity to small changes in camera viewpoint. Color coherence vector (CCV) stores the number of coherent versus incoherent pixels with each color. By separating coherent pixels from incoherent pixels, CCV's provide finer distinctions than color histograms. CCV's can be computed at over 5 images per second on a standard workstation.

3) Local Binary Pattern (LBP) Given a pixel in the input image, LBP [19] is computed by comparing it with its neighbors:

$L B P_{N, R}=\sum_{n=0}^{n-1} s\left(v_{n}-v_{c}\right) 2^{n}, s(x)=\left\{\begin{array}{l}1, x \geq 0 \\ 0, x<0\end{array}\right.$

Where, $v_{c}$ is the value of the central pixel, $v_{n}$ is the value of its neighbors, $\mathrm{R}$ is the radius of the neighborhood and $\mathrm{N}$ is the total number of neighbors. Suppose the coordinate of $v_{c}$ is $(0,0)$, then the coordinates of $v_{n}$ are $(R \cos (2 \pi n / N), R \sin (2 \pi n$ $/ N)$ ) . The values of neighbors those are not present in the 
image grids may be estimated by interpolation. Let the size of image is I*J. After the LBP code of each pixel is computed, a histogram is created to represent the texture image:

$$
\begin{aligned}
H(k)=\sum_{i=1}^{l} \sum_{j=1}^{J} f\left(L B P_{N, R}(i, j), k\right), k \in[0, K], \\
f(x, y)=\left\{\begin{array}{l}
1, x=y \\
0, \text { otherwise }
\end{array}\right.
\end{aligned}
$$

Where, $\mathrm{K}$ is the maximal LBP code value. In this experiment

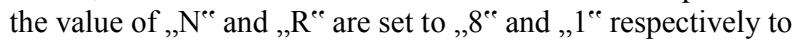
compute the LBP feature.

4) GLCM Features We use some global measures: mean $(\mu)$, contrast $(\mathrm{Cn})$, homogeneity $(\mathrm{Hg})$, energy $(\mathrm{En})$, variance $(\sigma 2)$, correlation (Cr), and entropy (Hn) [5]. We use following 8 GLCM features to train the neural network.

Mean:

$$
\mu_{i}=\sum_{i, j=0}^{N-1} i\left(P_{i, j}\right), \mu_{j}=\sum_{i, j=0}^{N-1} j\left(P_{i, j}\right)
$$

Contrast:

$$
\sum_{i, j=0}^{N-1} P_{i, j}(i-j)^{2}
$$

Homogeneity:

$\sum_{i, j=0}^{N-1} \frac{P_{i, j}}{1+(i-j)^{2}}$

Energy:

$$
\sum_{i, j=0}^{N-1} P_{i, j}^{2}
$$

Variance:

$\sigma_{i}^{2}=\sum_{i, j=0}^{N-1} P_{i, j}\left(i-\mu_{i}\right)^{2}, \sigma_{j}^{2}=\sum_{i, j=0}^{N-1} P_{i, j}\left(j-\mu_{j}\right)^{2}$

Correlation:

$$
\sum_{i, j=0}^{N-1} P_{i, j}\left[\frac{\left(i-\mu_{i}\right)\left(j-\mu_{j}\right)}{\sqrt{\left(\sigma_{i}^{2}\right)\left(\sigma_{j}^{2}\right)}}\right]
$$

Entropy:

$$
\sum_{i, j=0}^{N-1} P_{i, j}\left(-\ln P_{i, j}\right)
$$

Standard Deviation:

$$
\sigma_{i}=\sqrt{\sigma_{i}^{2}}, \quad \sigma_{j}=\sqrt{\sigma_{j}^{2}}
$$

We calculate values of these features for all the images in dataset. We create Excel Sheet of all these values and use this
Excel Sheet is as input to train the Probabilistic Neural Network.

\section{RESULTS AND DISCUSSION}

In this section we describe the classification results on our dataset. In our dataset we considered 10 types of diseased fruit classes namely Apple, Mango, Plum, Pear, Papaya, Banana, Orange, Pomegranate, Citrus limetta, Sapota. From each class we have collected 250 samples and totally 2500 images are used for experimentation. For classification we have used Artificial Neural Network.
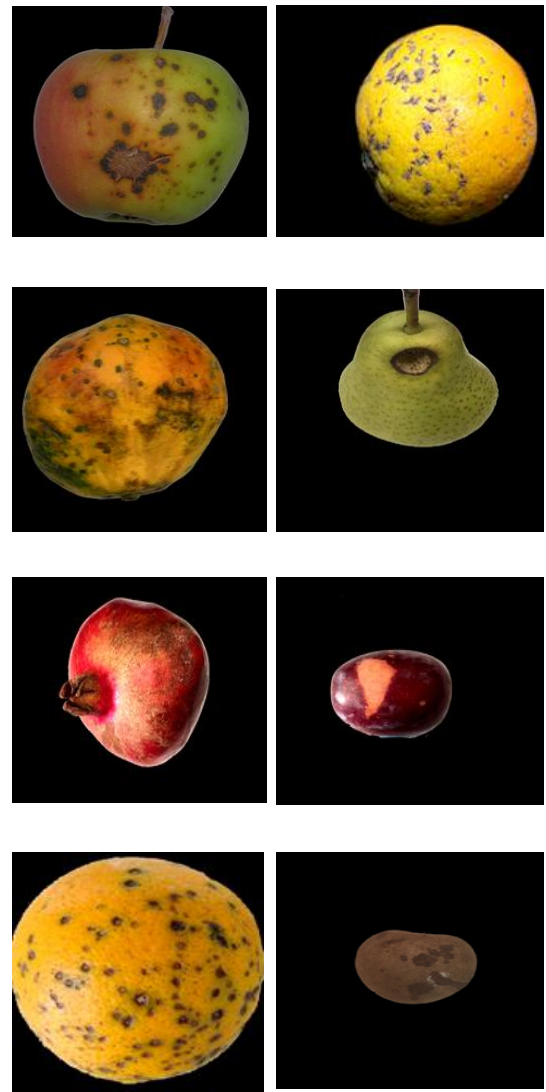

Figure 2: Our database sample fruit images.

In Table 1. shows that how the proposed method gives better results and we have presented result for both after segmentation(AS) and before segmentation(BS), the results of AS is better compared to BS for average classification for different training samples.

Table 1. Proposed method performance evaluation on classification for Before Segmentation (BS) and After Segmentation (AS).

\begin{tabular}{|c|c|c|c|c|c|c|}
\hline \multicolumn{2}{|c|}{ Proposed Method (PNN) } & \multicolumn{2}{c|}{$\begin{array}{c}\text { SVM } \\
\text { classifier }\end{array}$} & \multicolumn{2}{c|}{$\begin{array}{c}\text { KNN } \\
\text { classifier }\end{array}$} \\
\hline $\begin{array}{c}\text { Training } \\
\text { Samples }\end{array}$ & BS & AS & BS & AS & BS & AS \\
\hline $\mathbf{8 0}$ & 0.79 & 0.92 & 0.72 & 0.82 & 0.58 & 0.68 \\
\hline $\mathbf{5 0}$ & 0.72 & 0.85 & 0.54 & 0.68 & 0.52 & 0.64 \\
\hline $\mathbf{2 0}$ & 0.56 & 0.66 & 0.48 & 0.52 & 0.45 & 0.51 \\
\hline
\end{tabular}




\section{CONCLUSION}

In this paper, we have proposed method to classify fruit as diseased and non-diseased. Firstly, we used $\mathrm{K}$ means clustering method for segmentation of diseased regions. Later, we used to extract texture features on segmented diseased regions. We have conducted extensive experimentation and results shows that proposed method gives better performance with Probabilistic Neural Network compared to traditional classifiers.

\section{REFERENCES}

[1] Shiv Ram Dubey, Pushkar Dixit, Nishant Singh, Jay Prakash Gupta, 2013, “ Infected Fruit Part Detection using K-Means Clustering Segmentation Technique”, International Journal of Artificial Intelligence and Interactive Multimedia, Vol.2, , Page(s): 65-72 .

[2] A. Rocha, C. Hauagge, J. Wainer, and D. Siome, 2010 "Automatic fruit and vegetable classification from images," Computers and Electronics in Agriculture, Elsevier; vol. 70,. Page(s): 96-104.

[3] R. Gonzalez, R. Woods, Digital Image Processing, 3rd ed., Prentice-Hall, 2007.

[4] Bongani Malinga, Daniela Raicu, Jacob Furst, “ Local vs. Global Histogram-Based Color Image Clustering”

[5] T. Ojala, M. Pietikäinen, and T. T. Mäenpää, "Multiresolution gray-scale and rotation invariant texture classification with Local Binary Pattern," IEEE Trans. on Pattern Analysis and Machine Intelligence, vol. 24, no. 7 , pp. 971-987, 2002.

[6] Q. Li, M. Wang and W. Gu, "Computer Vision Based System for Apple Surface Defect Detection," Computers and Electronics in Agriculture, vol. 36, pp. 215-223, Nov. 2002.

[7] P. M. Mehl, K. Chao, M. Kim and Y. R. Chen, "Detection of Defects on Selected Apple Cultivars using Hyperspectral and Multispectral Image Analysis," Applied Engineering in Agriculture, vol. 18, pp. 219226, 2002.

[8] M. S. Kim, A. M. Lefcourt, Y. R. Chen and Y. Tao, "Automated Detection of Fecal Contamination of Apples Based on Multispectral Fluorescence Image Fusion," Journal of food engineering, vol. 71, pp. 85-91, 2005.

[9] O. Kleynen, V. Leemans and M. F. Destain, "Development of a Multi- Spectral Vision System for the Detection of Defects on Apples," Journal of Food Engineering, vol. 69, pp. 41-49, 2005.

[10] T. Ojala, M. Pietikäinen and T. T. Mäenpää, "Multiresolution Gray-Scale and Rotation Invariant Texture Classification with Local Binary Pattern," IEEE Transactions on Pattern Analysis and Machine Intelligence (TPAMI), vol. 24, no. 7, pp. 971-987, 2002.

[11] C. Bravo, D. Moshou, R. Oberti, J. West, A. McCartney, L. Bodria and H. Ramon, "Foliar Disease Detection in the Field using Optical Sensor Fusion," Agricultural Engineering International: the CIGR Journal of Scientific Research and Development, vol. 6, pp. 1-14, December 2004.

[12] D. Moshou, C. Bravo, R. Oberti, J. West, L. Bodria, A. McCartney and H. Ramon, "Plant Disease Detection Based on Data Fusion of Hyper-Spectral and Multi-
Spectral Fluorescence Imaging using Kohonen Maps," Real-Time Imaging, vol. 11, no. 2, pp. 75-83, 2005.

[13] L. Chaerle, S. Lenk, D. Hagenbeek, C. Buschmann and D. V. D. Straeten, "Multicolor Fluorescence Imaging for Early Detection of the Hypersensitive Reaction to Tobacco Mosaic Virus," Journal of Plant Physiology, vol. 164, no. 3, 253-262, 2007.

[14] D. Moshou, C. Bravo, S. Wahlen, J. West, A. McCartney and J. De Baerdemaeker, H. Ramon, "Simultaneous Identification of Plant Stresses and Diseases in Arable Crops using Proximal Optical Sensing and SelfOrganising Maps," Precision Agriculture, vol. 7, no. 3, pp. 149-164, 2006.

[15] H. Z. M. Shafri and N. Hamdan, "Hyperspectral Imagery for Mapping Disease Infection in Oil Palm Plantation using Vegetation Indices and Red Edge Techniques," American Journal of Applied Sciences, vol. 6, no. 6, pp. 1031-1035, 2009.

[16] J. Qin, F. Burks, M. A. Ritenour and W. G. Bonn, "Detection of Citrus Canker using Hyper-Spectral Reflectance Imaging with Spectral Information Divergence," Journal of Food Engineering, vol. 93, no. 2, pp. 183-191, 2009.

[17] F. Spinelli, M. Noferini and G. Costa, "Near Infrared Spectroscopy (NIRs): Perspective of Fire Blight Detection in Asymptomatic Plant Material," Proc. 10th International Workshop on Fire Blight, Acta Horticulturae 704, 2006, pp. 87-90.

[18] D. E. Purcell, M.G. O'shea, R. A. Johnson and S. Kokot, "Near-Infrared Spectroscopy for the Prediction of Disease Rating for Fiji Leaf Gall in Sugarcane Clones,' Applied Spectroscopy, vol. 63, no.4, pp. 450-457, 2009.

[19] L. G. Marcassa, M. C. G. Gasparoto, J. Belasque Junior, E. C. Lins, F. Dias Nunes and V. S. Bagnato, "Fluorescence Spectroscopy Applied to Orange Trees," Laser Physics, vol. 16, no. 5, pp. 884-888, 2006.

[20] L. Belasque, M. C. G. Gasparoto and L. G. Marcassa, "Detection of Mechanical and Disease Stresses in Citrus Plants by Fluorescence Spectroscopy," Applied Optics, vol. 7, no. 11, pp. 1922-1926, 2008

[21] E. C. Lins, J. Belasque Junior and L. G. Marcassa, "Detection of Citrus Canker in Citrus Plants using Laser Induced Fluorescence Spectroscopy," Precision Agriculture, vol. 10, pp. 319-330, 2009. International Research Journal of Engineering and Technology (IRJET) e-ISSN: 2395 -0056 Volume: 03 Issue: 03 | Mar-2016 www.irjet.net p-ISSN: 2395-0072 (C) 2016, IRJET | Impact Factor value: 4.45 | ISO 9001:2008 Certified Journal | Page 729

[22] C. M. Yang, C. H. Cheng and R. K. Chen, "Changes in Spectral Characteristics of Rice Canopy Infested with Brown Planthopper and Leaffolder," Crop Science, vol. 47, pp. 329-335, 2007.

[23] S. Delalieux, J. van Aardt, W. Keulemans, E. Schrevens and P. Coppin, "Detection of Biotic Stress (Venturia Inaequalis) in Apple Trees using Hyper-Spectral Data: Non-Parametric Statistical Approaches and Physiological Implications," European Journal of Agronomy, vol. 27, no. 1, pp. 130-143, 2007.

[24] B. Chen, K. Wang, S. Li, J. Wang, J. Bai, C. Xiao and J. 
International Journal of Computer Applications (0975 - 8887)

Volume 178 - No. 49, September 2019

Lai, "Spectrum Characteristics of Cotton Canopy Infected with Verticillium Wilt and Inversion of Severity Level," Computer and Computing Technologies in Agriculture, vol. 2, pp. 1169-1180, 2008.

[25] Y. H. Choi, E. C. Tapias, H. K. Kim, A. W. M. Lefeber, C. Erkelens, J. T. J. Verhoeven, J. Brzin, J. Zel and R. Verpoorte, "Metabolic Discrimination of Catharanthus Roseus Leaves Infected by Phytoplasma using 1H-NMR Spectroscopy and Multivariate Data Analysis," Plant
Physiology, vol. 135, pp. 2398-2410, 2004.

[26] F. Hahn, "Actual Pathogen Detection: Sensors and Algorithms-A Review," Algorithms, vol. 2, no. 1, pp. 301-338, 2009

[27] S. Sankarana, A. Mishraa, R. Ehsania and C. Davisb, "A Review of Advanced Techniques for Detecting Plant Diseases" Computers and Electronics in Agriculture, vol. 72, pp. 1-13, 2010 